\title{
ASPECTOS EMOCIONAIS E LUTO NAS TENTATIVAS FRUSTRADAS EM REPRODUÇÃO HUMANA ASSISTIDA: UMA REVISÃO DA LITERATURA
}

\author{
Thalyta Freitas dos Santos Laguna1; Márcia Elisa Jaeger²
}

\section{RESUMO}

O objetivo deste trabalho foi identificar se a literatura compreende o luto como um estado emocional legítimo nos processos de reprodução humana assistida (RHA), a partir de uma revisão da literatura. A busca foi realizada em livros impressos e nas plataformas SCIELO, Google Acadêmico e BVS. Incluiu-se para análise 16 estudos dos últimos 5 anos (2016-2021). Os resultados apontam que há diversos aspectos emocionais que emergem durante o processo de RHA, incluindo sentimentos negativos e de descrença. Algumas estratégias de enfrentamento como a resolução de problemas, bem como intervenções terapêuticas variadas tem-se mostrado eficazes no manejo das emoções provenientes dos processos de RHA. Os estudos indicaram que os casais que falham nos tratamentos de RHA possuem mais distúrbios emocionais, incluindo o luto, que embora seja um estado emocional que pode surgir durante o tratamento, percebeu-se não ser reconhecido como legítimo na sociedade.

Palavras-chave: Estresse Emocional; Infertilidade; Técnicas de Reprodução.

Eixo Temático: Atenção Integral e Promoção à Saúde (AIPS).

\section{INTRODUÇÃO}

Segundo a Organização Mundial da Saúde (WHO, 2016), a infertilidade é a dificuldade de um casal conseguir a gravidez no intervalo de um período de 12 meses tendo relações sexuais frequentes, sem qualquer uso de contraceptivos. Também se considera infertilidade as dificuldades consecutivas de uma mulher em manter a gravidez. São várias as causas que podem gerar infertilidade tanto em mulheres quanto em homens e estima-se que ela pode afetar cerca de $10 \%$ a $20 \%$ dos casais em idade reprodutiva, independente de suas origens étnicas ou sociais (WHO, 2016).

\footnotetext{
1 Universidade Franciscana - UFN. E-mail: thalytalaguna@gmail.com

2 Universidade Franciscana - UFN. E-mail: marcia.jager@ufn.edu.br
} 
Há no mínimo cinco décadas, a humanidade testemunha grandes avanços na ciência em diversos âmbitos, incluindo o da saúde reprodutiva. Nos últimos 40 anos, estima-se que mais de 8 milhões de bebês nasceram através das Técnicas de Reprodução Humana Assistida (TRA). Em 1978 quando o primeiro "bebê de proveta" veio ao mundo, na Inglaterra, a chance de concepção por meio de Fertilização In Vitro (FIV) era de 5\%. Atualmente, calcula-se que a chance média seja de $40 \%$ até $50 \%$ (BADALOTTI, HENTSCHKE, PETRACCO, 2019). Especialmente no Brasil, em 1984, quando a primeira criança veio ao mundo através da técnica de FIV, a premissa inicial para utilização de tal procedimento era de que as mulheres com infertilidade necessariamente deveriam ter problemas tubários. Com o passar dos anos, as técnicas tornaram-se mais conhecidas, abrindo assim um leque de indicações para RHA. Desta forma, compreende-se que este é um método que tem possibilitado à muitas pessoas - independente da configuração familiar - tornarem-se mães e pais (MELAMED, DORNELLES, 2019).

Félis e Almeida (2016) destacam que a inabilidade de gerar um filho pode ser catastrófica no que tange aos aspectos emocionais dos envolvidos, uma vez que atingem perspectivas pessoais, sociais e até religiosas, causando emoções associadas à perda, à falha - sensação de que não conseguiu ser mãe e pai - e até à exclusão na sociedade, pela falta de filhos. Estes autores indicam que quando estas emoções são vivenciadas de maneira disfuncional, elas podem culminar em quadros psicopatológicos associados aos Transtornos do Humor (APA, 2014), além de sentimentos intensos de raiva e desvalorização pessoal e na autoestima. Batista, Bretones e Almeida (2016) aludem que, além dos prejuízos que a dificuldade de engravidar e a perda de um bebê podem causar à saúde mental, outros aspectos da vida dos envolvidos podem ser afetados negativamente, tais como a vida pessoal, conjugal e profissional gerando tensão, ansiedade, isolamento e culpa.

Casais que vivenciam a infertilidade deparam-se com a realidade de que a biologia da reprodução humana não é fácil, direta e linear: vai além da escolha de um par, da relação sexual, da gestação e do parto. Deparar-se com a infertilidade pode desencadear antigos traumas e sentimentos de inadequação, em meio a um processo marcado - na maioria das vezes - por longos períodos de interação 
GDUCAÇÃO, SAÚDE

ETECNOLOGIA

26 A 28 DE OUTUBRO DE 2021

médica (FARINATI, RIGONI, MÜLLER, 2006). Conquanto, apesar dos benefícios trazidos pela tecnologia da medicina reprodutiva, a utilização destas técnicas não garante a gestação e a concepção. Ainda assim, os tentantes criam uma expectativa de êxito nos tratamentos, porém a possibilidade de insucesso, apesar de abordada pelos profissionais envolvidos no processo, é pouco aprofundada e debatida por eles (MELAMED, DORNELLES, 2019).

Vives et al. (2019) reforçam que nem todos os tentantes que se submetem a RHA obtém o resultado esperado: o bebê em seu colo. Os autores destacam que o insucesso das tentativas pode gerar o sentimento de falha e culminar no luto, um processo funcional, adaptativo e necessário para a elaboração de qualquer perda. Entretanto, os prejuízos relacionados às falhas na RHA são vistos como ambivalentes, ou seja, caracterizados pela falta de clareza no que tange ao que foi perdido, o que se perdeu e se de fato houve perda ou não, podendo gerar diversas consequências psicológicas negativas nos envolvidos. Isso posto, o luto proveniente de tentativas de RHA sem sucesso pode gerar cognições e emoções ligadas ao fracasso, vazio, incompletude, tristeza e melancolia, especialmente quando não reconhecido e validado pela sociedade (SILVA et al., 2020).

Destarte, por meio de uma breve análise de estudos científicos realizados e publicados nos últimos 5 anos - 2016 a 2021 - este trabalho tem por objetivo identificar, a partir de uma revisão de literatura, se os autores compreendem o luto como um estado emocional legítimo nos processos de reprodução humana assistida.

\section{METODOLOGIA}

Este estudo é uma revisão da literatura realizada no período de fevereiro a setembro de 2021. Para embasar a pesquisa, utilizou-se a questão norteadora "O luto é compreendido como um dos estados emocionais dentro dos processos de reprodução humana assistida?".

A busca foi realizada em livros impressos e nas plataformas Scientific Eletronic Library Online (SCIELO), Google Acadêmico e Biblioteca Virtual em Saúde (BVS), nas quais utilizou-se os descritores: Técnicas de Reprodução, Infertilidade e 
DUCAÇÃO, SAÚDE

ETECNOLOGIA

26 A 28 DE OUTUBRO DE 2021

Emocional. Considerou-se as bibliografias dos últimos 5 anos, incluindo-se estudos em português e inglês que abordaram o tema proposto. Excluiu-se do estudo materiais duplicados, teses e dissertações, bem como, trabalhos que não respondiam à questão norteadora.

Uma vez elencados os critérios de inclusão/exclusão, selecionou-se previamente 28 trabalhos e após a leitura do texto completo, excluíram-se 12 materiais, sendo incluídos para análise 16 estudos.

\section{RESULTADOS E DISCUSSÕES}

A análise dos materiais teve como principal objetivo responder à questão norteadora do estudo e, desta forma, para que isso acontecesse, o trabalho foi dividido em três categorias temáticas: (1) Aspectos emocionais em RHA; (2) As emoções e as falhas no tratamento: luto? e (3) Estratégias de Enfrentamento e Intervenções terapêuticas.

\subsection{ASPECTOS EMOCIONAIS EM RHA}

Em estudo sobre a perspectiva de casais em relação à infertilidade, Félis e Almeida (2016) apontam que essa condição pode interromper projetos de vida pessoais e do casal e causar intenso sofrimento psíquico, visto que para algumas pessoas, ter um filho é um dos principais objetivos de vida e a impossibilidade de gerá-lo culmina em intenso sofrimento. A opção pelas tecnologias de RHA tende a gerar um desgaste não somente financeiro, mas também emocional, uma vez que vem permeado pela notícia do diagnóstico de infertilidade, que carrega consigo grande potencial de resultados psicossociais negativos para a saúde mental (BATISTA, BRETONES, ALMEIDA, 2016;). Destarte, alguns estudos encontrados para composição desta revisão reportam que os Transtornos Depressivos Maiores e os Transtornos de Ansiedade estão diretamente associados como causa e/ou consequência da infertilidade.

Dois dos estudos analisados tinham como principal objetivo avaliar as reações emocionais de mulheres e homens durante o processo de FIV, a fim de identificar possíveis diferenças entre eles. Como resultado compreendeu-se que, 
embora ambos experimentassem angústia e sofrimento durante RHA, as mulheres apresentavam maiores níveis de ansiedade e depressão antes e durante o tratamento, especialmente no dia da retirada dos oócitos, antes, durante e depois da transferência dos embriões, bem como na espera para realização do teste de gravidez. Já os homens apresentavam elevado score de depressão antes do tratamento, que também aumentava durante a espera do teste de gravidez (YING, WU, LOKE, 2016; MOURA-RAMOS et al., 2016).

A fim de realizar o sonho da maternidade, muitas mulheres diagnosticadas inférteis submetem-se à vários procedimentos invasivos e nem sempre confortáveis, como uso de medicamentos orais, aplicação de injeções e exames variados. Assim, apesar de oferecer a chance de conceber, os procedimentos de RHA tendem a gerar elevado sofrimento psicológico às mulheres e ao casal (CASU, GREMIGNI, 2016;). Além disso, outro estudo que objetivava comparar se o modo de concepção - espontâneo ou com auxílio de TRA - têm efeito sobre os níveis de ansiedade e depressão, destacou que casais que concebem um ou mais de um bebê espontaneamente apresentam menos riscos de aumento de ansiedade e depressão durante a gravidez, parto e pós-parto, enquanto os pais que conceberam com o auxílio da RHA apresentaram esses sintomas clinicamente significativos, além de poderem apresentar mais dificuldades de adaptação ao período perinatal (TENDAIS, FIGUEIREDO, 2016).

Um estudo brasileiro analisou mulheres grávidas por meio de RHA, tanto em primeiro tratamento quanto aquelas que passaram por vários ciclos malsucedidos, com o intuito de verificar se a experiência da gravidez foi ou não afetada pelos processos de RHA. Os resultados levantados pelos autores sugeriram que, para as mulheres participantes do estudo, a gravidez era considerada uma recompensa pelas dificuldades enfrentadas durante toda a trajetória de tratamentos, por mais que tenham apresentado sintomas depressivos e manifestado emoções e sentimentos disfuncionais. Entretanto, observou-se que as que engravidaram no primeiro ciclo de procedimentos tiveram menos impactos emocionais e psicológicos do que aquelas que passaram por ciclos malsucedidos (DORNELLES et al., 2016). 
Isso posto, compreende-se que o estresse, a ansiedade e a angústia geradas pela infertilidade podem promover grande impacto não só na saúde física, mas também na saúde mental, uma vez que em ambos os sexos a infertilidade pode ser considerada uma experiência devastadora. Porém, ainda maior para as mulheres, visto o grau de importância social e cultural atribuídas a identidade feminina. Outrossim, quando o filho sonhado não se torna real, sentimentos de incompletude podem culminar em um tipo de luto que ocupa um não lugar: o luto da infertilidade (SILVA et al., 2020).

\subsection{AS EMOÇÕES E AS FALHAS NO TRATAMENTO: LUTO?}

De acordo com Silva et al. (2020), quando o filho sonhado não se torna real mesmo depois de todas as tentativas em RHA - interrompidas seja por incapacidade física ou financeira - diversas consequências podem ser geradas em várias esferas da vida. Para as autoras, a expectativa de ter um filho é carregada de muitos significados, representando muitas vezes uma nova vida, com novos começos e reinvenções.

Bhat e Byatt (2016) afirmam que tanto a infertilidade quanto as perdas perinatais e os insucessos nos tratamentos de RHA podem ser considerados como traumas reprodutivos que são capazes de influenciar a percepção que a mulher tem de si mesma e ser uma relevante fonte de estresse que pode gerar importantes consequências psicológicas. Silva et al. (2020) destacam que o luto da infertilidade ocupa um não lugar, ou seja, um luto desconhecido, visto que, para a sociedade, não há clareza sobre o que foi perdido. Inclusive, os autores argumentam que duvida-se que os insucessos da RHA sejam considerados perdas, uma vez que ocorreram antes da existência física de uma criança.

O luto é uma etapa necessária na elaboração de qualquer perda; é considerado ambivalente quando é caracterizado pela falta de clareza com relação ao que foi perdido, o que se perdeu e se houve perda ou não, podendo gerar diversas consequências negativas nos envolvidos. A vista disso, alguns estudos apontam que o luto das tentativas falhadas não é legitimamente debatido, desconsiderando-se, nesse caso, que a perda da possibilidade de ter um filho pode 
gerar igualmente, tristeza, fracasso, vazio e sentimento de incompletude (BOER, BONDEVIK, SOLBRAEKKE, 2020; SILVA et al., 2020).

Gramacho (2021) aponta que as perdas não resolvidas são consideradas critérios de postergação e de exclusão do tratamento de RHA. A autora indica que são perdas não reconhecidas dentro dos "critérios do luto" estabelecidos por uma sociedade que especifica quem, quando, como, onde e por quanto tempo podemos e devemos expressar sentimentos de pesar.

Assim, a partir dos estudos revisados, compreende-se que o direito de vivenciar o luto é retirado pela sociedade, condenando os tentantes de colos vazios ao isolamento silencioso e à falta de amparo, incrementados pelo sentimento de alienação e solidão. Destarte, são necessárias algumas estratégias de enfrentamento e intervenções terapêuticas que auxiliem as pessoas inférteis a superar a difícil realidade do insucesso na RHA.

\subsection{ESTRATÉGIAS DE ENFRENTAMENTO E INTERVENÇÕES TERAPÊUTICAS}

Os processos de RHA podem ser considerados importantes caminhos para as pessoas inférteis, porém podem ser percebidos pelos tentantes como fontes de ansiedade e estresse, devido ao excesso de etapas médicas vivenciadas, atreladas ao elevado custo financeiro. Os estudos analisados apontaram algumas estratégias de enfrentamento utilizadas pelos tentantes para manejar o turbilhão de emoções, a ansiedade e depressão iminentes. Percebeu-se em alguns estudos revisados que uma das estratégias comumente utilizadas pelos profissionais junto com os tentantes é a de resolução de problemas, a fim de auxiliar no controle de pensamentos, sentimentos, ações e emoções diante de situações estressantes. Os dados evidenciam que uma vez adquirida a capacidade de resolver problemas, os sentimentos de esperança e as emoções positivas podem auxiliar a diminuir a ansiedade e depressão comum aos tentantes (GOJANI et al., 2017; GHASEMI et al., 2017; NAGÓRSKA et al., 2019).

Diante dos sintomas apresentados pelos casais submetidos à RHA, percebeu-se que as principais intervenções realizadas são na abordagem cognitivo comportamental - através de psicoterapia individual e de grupo. Alguns estudos 
EUGAÇÃO, SAÚDE

ETECNOLOGIA

26 A 28 DE OUTUBRO DE 2021

trouxeram também as terapias complementares tais como técnicas de meditação, relaxamento e mindfulness durante os processos de FIV, constatando que podem ser ferramentas potentes na redução de ansiedade, podendo proporcionar uma experiência mais positiva (MASOUMI et al., 2019; LOGIUDICE, MASSARO, 2018).

\section{CONCLUSÃO}

A partir da revisão realizada verificou-se um consenso acerca dos impactos psicológicos que os processos em RHA podem causar nos envolvidos. As diversas tentativas de concepção podem gerar intensas emoções nas pessoas tentantes, incluindo as associadas à frustração e impotência e ainda, o desenvolvimento de um conjunto de sintomas ligados às cognições, emoções e comportamentos disfuncionais que podem culminar em estados psicológicos de ansiedade e até depressão. Os estudos revisados ainda indicaram que os casais que não tem sucesso nos tratamentos de RHA possuem mais distúrbios emocionais do que os que obtiveram sucesso. Entretanto, apesar de o luto proveniente do insucesso em RHA ser compreendido como um aspecto emocional, ainda não é plenamente reconhecido nem validado pela sociedade, uma vez que se tratam de perdas ambíguas, nas quais não há clareza com relação ao que foi perdido, o que se perdeu e ainda se houve perda ou não. Por fim, compreende-se a relevância do tema e sugere-se a pesquisa e desenvolvimento de estudos voltados para o luto proveniente da infertilidade.

\section{REFERÊNCIAS}

\section{AMERICAN PSYCHIATRIC ASSOCIATION - APA. Manual diagnóstico e} estatístico de transtornos mentais: DSM-5. Porto Alegre: Artmed, 2014.

BADALOTTI, Mariangela; HENTSCHKE, Marta Ribeiro; PETRACCO, Alvaro. Diretrizes e possibilidades da medicina em reprodução humana assistida. IN: QUAYLE, Julieta; DORNELLES, Lia Mara Netto; FARINATI, Débora Marcondes. Psicologia em reprodução assistida. São Paulo: Editora dos editores, p. 3-29. 2019. 
BHAT, Amritha; BYATT, Nancy. Infertility and Perinatal Loss: When the Bough Breaks. Curr Psychiatry Rep. V. 18(3). Disponível em: https://pubmed.ncbi.nlm.nih.gov/26847216/

BOER, Marjolein Lotte de; BONDEVIK, Hilde; SOLBRAEKKE, Kari Nyheim. Beyond pathology: women's lived experiences of melancholy and mourning in infertility treatment. Medical Humanities, V. 46, 214-225, 2020. Disponível em: https://mh.bmj.com/content/46/3/214

BATISTA, Luiz Augusto Teixeira; BRETONES, Wagner Henrique Daibert; ALMEIDA, Rogério José de. O impacto da infertilidade: narrativas de mulheres com sucessivas negativas pelo tratamento de reprodução assistida. Reprodução e Climatério, Goiás, $\quad$ v. 31(3), 121-127, $2016 . \quad$ Disponível em: https://www.sciencedirect.com/science/article/pii/S1413208716300292

CASU, Giulia; GREMIGNI, Paola. Screening for infertility-related stress at the time of initial infertility consultation: psychometric properties of a brief measure. J Adv Nurs, V. 72(3), 693-706, 2016. Disponível em: https://pubmed.ncbi.nlm.nih.gov/26466927/

DORNELLES, Luciano et al. The experience of pregnancy resulting from Assisted Reproductive Technology (ART) treatment: A qualitative Brazilian study. Women Birth, V. 29(2), 123-127, 2016. Disponível em: https://pubmed.ncbi.nlm.nih.gov/26388215/

FARINATI, Débora Marcondes; RIGONI, Maísa dos Santos; MÜLLER, Marisa Campio. Infertilidade: um novo campo da Psicologia da saúde. Estudos de Psicologia, Campinas, v. 23(4), 433-439, 2006. Disponível em: http://pepsic.bvsalud.org/pdf/epc/v23n4/v23n4a11.pdf 
FÉLIS, Keila Cristina; ALMEIDA, Rogério José de. Perspectiva de casais em relação à infertilidade e reprodução assistida: uma revisão sistemática. Reprodclim., Goiás, v.31(2), 105-111, $2016 . \quad$ Disponível em: https://reader.elsevier.com/reader/sd/pii/S1413208716000078?token=D8078131F55 FAE47D40ECC05BA24A694ABBFC8F9BE9618E36E6FE28D62B44B7EE5ED167E 6303570D95D3AF077EC5CAD5\&originRegion=us-east1\&originCreation $=20210920001813$

GHASEMI, Marzieh et al. The effect of a positive reappraisal coping intervention and problem-solving skills training on coping strategies during waiting period of IUI treatment: An RCT. Int J Reprod Biomed. V. 5(11), 687-696. 2017.

GOJANI, Marziyeh Ghasemi et al. The effect of problem-solving skill training on mental health and the success of treatment of infertile women under intrauterine insemination treatment. J Edu Health Promot,V.6, 107. 2017. Disponível em: https://www.jehp.net/article.asp?issn=2277-

9531; year=2017; volume=6;issue=1; spage=107; epage=107; aulast=Gojani

GRAMACHO, Patrícia Marinho. Seria o luto uma contraindicação à Reprodução Assistida? Instituto Suassuna, 2021. Disponível em: https://www.institutosuassuna.com.br/seria-o-luto-uma-contraindicacao-areproducao-assistida/

LOGIUDICE, Jenna; MASSARO, Jenna. The impact of complementary therapies on psychosocial factors in women undergoing in vitro fertilization (IVF): A systematic literature review. Appl Nurs Res. V. 39, 220-228. 2017. Disponível em: https://pubmed.ncbi.nlm.nih.gov/29422163/

MASOUMI, Seyedeh Zahra et al. What Psychiatric Interventions Are Used for Anxiety Disorders in Infertile Couples? A Systematic Review Study. Iran J Psychiatry. V. 14(2),160-170, 2019. Disponível em: https://pubmed.ncbi.nlm.nih.gov/31440298/ 
MELAMED, Rose Marie Massaro; DORNELLES, Lia Mara Netto. Desenvolvimento da reprodução assistida: O viés da psicologia. IN: QUAYLE, Julieta; DORNELLES, Lia Mara Netto; FARINATI, Débora Marcondes. Psicologia em reprodução assistida. São Paulo: Editora dos editores, p. 31-41. 2019.

MOURA-RAMOS, Mariana et al. Does infertility history affect the emotional adjustment of couples undergoing assisted reproduction? the mediating role of the importance of parenthood. Br J Health Psychol, V. 21(2), 302-17. 2016. Disponível em: https://pubmed.ncbi.nlm.nih.gov/27059275/

NAGÓRSKA, Małgorzata Nagórska et al. Gender Differences in the Experience of Infertility Concerning Polish Couples: Preliminary Research. Int J Environ Res Public Health.

V. 2;16(13), 2337. 2019. Disponível em: https://pubmed.ncbi.nlm.nih.gov/31269703/

SILVA, Eliane Souza Ferreira da. et al. In: CASSELATO, Gabriela (Org.). Luto por perdas não legitimadas na atualidade. São Paulo: Summus, p.215-230. 2020.

TENDAIS, Iva; FIGUEIREDO, Bárbara. Parents' anxiety and depression symptoms after successful infertility treatment and spontaneous conception: does singleton/twin pregnancy matter? Hum Reprod, V. 31(10), 2303-12. 2016. Disponível em: https://pubmed.ncbi.nlm.nih.gov/27609986/

VIVES, Renata Viola et al. A infertilidade como fantasma da reprodução assistida: suas implicações na subjetividade do sujeito. In: VIVES, Renata Viola. (Org.). Reflexões Psicanalíticas sobre reprodução humana assistida. Porto Alegre: Sulina, p. 151-164. 2019.

WORLD HEALTH ORGANIZATION. World Bank Special Programme of Research, Development and Research Training in Human Reproduction (HRP). Annual 
EDUCAÇÃO, SAÚDE

ETECNOLOGIA

26 A 28 DE OUTUBRO DE 2021

Technical Report

2015.

Geneva,

2016.

Disponível

em:

http://apps.who.int/iris/bitstream/handle/10665/246092/WHO-RHR-HRP-16.08eng.pdf?sequence $=1$

YING, Liying; WU, Lai Har; LOKE, Alice Yuen. Gender differences in emotional reactions to in vitro fertilization treatment: a systematic review. J Assist Reprod Genet, $\quad$ V. 33(2), 167-79. 2016. Disponível em: https://pubmed.ncbi.nlm.nih.gov/26712577/ 